\title{
CONTROLE DA PRÉ-CARGA EM PARAFUSOS: CORRELAÇÃO ENTRE O MÉTODO DO TORQUE E O ALONGAMENTO MEDIDO POR ULTRASSOM
}

Felipe Samuel Goettems ' Rubem Manoel de Braga ${ }^{2}$ Afonso Reguly ${ }^{2}$

\section{Resumo}

Este trabalho tem como principal objetivo comparar dois métodos de controle da pré-carga, ou seja, a tensão na

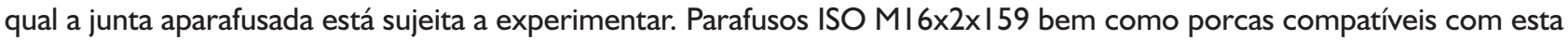
especificação de parafusos foram utilizados no sistema. O primeiro método é o controle desta tensão através da medição do torque com torquímetro e o segundo é o controle pela medição por ultrassom do alongamento. Os parafusos foram submetidos a torque conhecido por um torquímetro de estalo e em sua extremidade o seu comprimento foi monitorado por um transdutor ultrassônico. O dispositivo foi fixado dentro de uma célula de carga para monitorar a carga efetiva aplicada quando o parafuso fora submetido ao torque. A partir dos resultados obtidos foi possível comprovar, através de equações adequadas, que o controle feito por ultrassom é muito mais confiável e apresenta resultados de carga mais próximos dos valores de carga efetiva, sempre considerando tensões dentro do regime elástico do material.

Palavras-chave: Parafuso; Torque; Ultrassom; Alongamento.

\section{TIGHTENING LOAD CONTROL: CORRELATION BETWEEN TORQUE METHOD AND ULTRASONIC METHOD OF BOLT ELONGATION}

\begin{abstract}
This present work aims at comparing two methods of control of the load applied to ISO MI6 2 × I59 fasteners. In the first method, the control is performed by measuring torque with a manual torque wrench and the second, not yet widely used, the control is performed by measuring the resulting bolt elongation with ultrasound. The bolts were subjected to torque by a click torque wrench and at its other end, its length was monitored by an ultrasonic transducer. This whole device was fixed within a load cell, so that the applied load was monitored on the bolt. The main conclusion of this work is that the control performed by ultrasound is more reliable than the control done by the torque method, obtaining results very close to the load values of effective load, considering loads under elastic region.
\end{abstract}

Keywords: Fastener; Torque; Ultrasound; Elongation.

\section{INTRODUÇÃO}

A busca constante pela redução de custos na indústria metal-mecânica é uma realidade. Quanto maior for o controle do processo de fabricação menor serão as possibilidades de erros e isso pode acarretar em uma diminuição dos custos de manutenção ou substituição de peças. Em uniões parafusadas, tal comportamento não poderia ser diferente.
A fadiga é responsável por cerca de $85 \%$ das falhas em uniões parafusadas, sendo que deste percentual cerca de $45 \%$ está relacionado com erros de montagem [ 1$]$. Em termos do torque aplicado, aparentemente existe uma estreita faixa de valores com os quais aumentam a vida em fadiga de uma junta. É consenso que valores extremos são

'Programa de Pós-graduação em Engenharia de Minas, Metalúrgica e de Materiais - PPGE3M, Laboratório de Corrosão, Proteção e Reciclagem de Materiais - LACOR, Universidade Federal do Rio Grande do Sul - UFRGS, Porto Alegre, RS, Brasil. E-mail: felipegoettems@yahoo.com.br

${ }^{2}$ Programa de Pós-graduação em Engenharia de Minas, Metalúrgica e de Materiais - PPGE3M, Laboratório de Metalurgia Física - LAMEF, Universidade Federal do Rio Grande do Sul - UFRGS, Porto Alegre, RS, Brasil. 
prejudiciais. Apertos muito próximos ao limite de escoamento do material são danosos uma vez que ocorrerá naturalmente a aplicação de uma carga externa à junta, podendo facilmente o parafuso se deformar plasticamente. Valores de pré-carga próximos de zero também são prejudiciais, pois aumentam as chances da desmontagem bem como da fadiga de baixo ciclo [2]. Algumas citações remetem para valores da ordem de $90 \%$ da tensão de escoamento do material [3] sugerindo que nesta faixa aumenta-se a resistência do parafuso, porém somente em casos onde não haja solicitações de fadiga.

Um grande desafio da indústria que utiliza uniões parafusadas é a de garantir que no momento que o parafuso sofre o torque de aperto, a tensão ou pré-carga estipulada em projeto para a junta aparafusada seja de fato alcançada. Existem duas maneiras genéricas de se controlar esta pré-carga em parafusos, dentre outras menos utilizadas: controlando o torque aplicado e correlacionando este valor com a tensão através de tabelas ou controlando o alongamento do parafuso e transformando estes valores em tensão através de equações adequadas [4-6].

$\mathrm{Na}$ grande maioria dos casos o controle da pré-carga em parafusos de uso crítico como os usados em trocadores de calor é feito através do controle do torque, seja pelo método do torque ou pelo método do torque-ângulo. No método de aperto pelo torque, também conhecido como método do torque simples, geralmente define-se uma "janela de torque" alvo onde o equipamento responsável pela aplicação do torque deve chegar, e com isso "garante-se" uma força tensora capaz de sustentar a junta. Já no método do torque-ângulo, a primeira etapa do processo é semelhante ao método anterior, diferenciando um do outro pelo fato de que após se atingir um determinado torque, faz-se um controle do ângulo no qual o parafuso gira. Porém, estudos práticos demonstram que quando o controle da tensão é feito através de torquímetros manuais previamente calibrados, o erro associado pode chegar na casa de $20 \%$ [I]. Para casos mais críticos, torquímetros eletrônicos devidamente calibrados conseguem atingir erros menores que $5 \%[I]$, porém o custo envolvido na aquisição e calibração periódica destes equipamentos não é baixo.

Grande parte do erro na relação torque e força está associado ao fato de que o método não prevê a perda de uma parte da tensão resultante em tensões de torção do parafuso e também não considera a perda de carga devido ao atrito entre as partes envolvidas no aperto [3,7,8]. Estima-se que somente $10 \%$ do torque aplicado seja transformado em força, sendo os outros $50 \%$ gastos no atrito na região da cabeça do parafuso e outros $40 \%$ perdidos na região da rosca [3,4]. Também é consenso que a região buscada para a aplicação da força de aperto de um parafuso é sempre dentro da zona elástica, ou seja, região linear e controlada pela lei de Hooke $[3,4,9]$. Esta afirmação é válida e requerida tanto durante o processo de aperto do parafuso quanto após a exposição de cargas externas previamente calculadas.

Métodos mais confiáveis e simples para o controle da pré-carga em parafusos estão surgindo com grande potencial de aplicação na indústria e futuramente tendem a substituir os métodos tradicionais [9]. Um destes novos métodos é chamado de controle da tensão pelo alongamento, onde a pré-carga teria relação direta com o aumento do seu comprimento na medida em que o torque é aplicado. Este controle da variação no seu comprimento pode ser feito através de transdutores ultrassônicos e, com auxílio de equações pertinentes é possível converter essa variação em carga e depois em tensão $[4,10]$.

O presente trabalho tem como objetivo comparar o erro relativo à tensão efetiva aplicada no parafuso através de dois diferentes métodos. O primeiro método, tradicional e amplamente utilizado, foi o controle realizado através do torque utilizando-se um torquímetro de estalo previamente calibrado. O segundo método foi controlando-se o alongamento do parafuso em tempo real através de um transdutor ultrassônico e convertendo em tensão após aplicação de formulação adequada. A tensão efetiva aplicada no parafuso foi medida através de uma célula de carga previamente ajustada e calibrada.

\section{MATERIAIS E MÉTODOS}

Após um prévio estudo acerca da limitação dos equipamentos que seriam utilizados no ensaio foi selecionada uma amostragem de parafusos com as seguintes características: quatro parafusos ISO MI6, rosca métrica normal (passo de $2 \mathrm{~mm}$ ), comprimento nominal de $159 \mathrm{~mm}$ (sendo $44 \mathrm{~mm}$ de parte roscada), cabeça sextavada de $10 \mathrm{~mm}$ de altura e classe de resistência 5.8. A porca era compatível com o parafuso e possuía altura de $12,5 \mathrm{~mm}$, largura de $24 \mathrm{~mm}$ e classe 5 .

Os parafusos foram adquiridos de um lote aleatório comercial e passaram por uma bateria de testes no intuito de verificar se estavam de acordo com a norma ISO 898-I:20 I 3 [ I I] e se possuíam representatividade em termos de dureza e comprimento total, visto que estas são variantes críticas para a medição do alongamento através do ensaio de ultrassom. Cada um dos parafusos foi submetido então a uma breve caracterização metalúrgica composta por ensaio de dureza (Durômetro Brinell, marca Wolpert) e análise química (Espectrômetro de Emissão Óptica por Centelha, marca Spectro e modelo Spectrolab), e ainda pela medição do comprimento através de paquímetro, conforme Tabelas I e 2 .

O controle da carga efetiva foi realizado através de uma célula de carga (MTS série 66I.22D-0I com carga máxima de $250 \mathrm{kN}$ e erro de $0,15 \%$ do fundo de escala) e, para a aplicação do torque, um torquímetro tipo estalo (Armstrong, série 64.086 , com fundo de escala de $348,4 \mathrm{Nm}$ e tolerância de + ou - 3\%).

Para a medição do alongamento do parafuso, foi utilizado um aparelho de ultrassom convencional (Panametrics, modelo Epoch XT) juntamente com um transdutor normal

Tabela I. Resultados médios obtidos no ensaio de dureza e comprimento para os quatro parafusos ensaiados

\begin{tabular}{ccc}
\hline Parafuso & Dureza (HB) & Comprimento $(\mathrm{mm})$ \\
\hline 01 & 237 & 159,1 \\
02 & 229 & 158,9 \\
03 & 230 & 159,0 \\
04 & 235 & 158,8 \\
ISO 898-1:2013 [II] & $152-238$ & - \\
\hline
\end{tabular}


Tabela 2. Composição química dos parafusos ensaiados

\begin{tabular}{cccccc}
\hline Parafuso & $C(\%)$ & $M n(\%)$ & $S(\%)$ & $P(\%)$ & $S i(\%)$ \\
\hline 01 & 0,42 & 0,64 & 0,02 & 0,02 & 0,28 \\
02 & 0,38 & 0,66 & 0,02 & 0,02 & 0,31 \\
03 & 0,40 & 0,68 & 0,02 & 0,02 & 0,38 \\
04 & 0,41 & 0,66 & 0,02 & 0,02 & 0,36 \\
ISO 898-I:2013 [II] & $0,15-0,55$ & - & 0,06 máx. & 0,05 máx. & - \\
\hline
\end{tabular}

(Karl Deutsch, série KBA com freqüência de $10 \mathrm{Mhz}$, diâmetro de $9 \mathrm{~mm}$ e erro de medição estimado em 0,05mm).

Inicialmente, os parafusos foram montados sem folga no dispositivo dentro da célula de carga e o torquímetro foi acoplado à sua cabeça (Figura I). Na outra extremidade, o transdutor foi preso e calibrado com o comprimento inicial do parafuso.

Após a montagem de todo o aparato, a célula de carga foi zerada para que, a partir dali qualquer variação no torque indicaria consequente aumento da carga (ver Figura 2). Após a etapa de montagem, o parafuso foi submetido a torque aplicado através do torquímetro referido com valor inicial de 43,4 $\mathrm{Nm}$ e um aumento gradual conforme escala do torquímetro até um valor de 165,4 Nm. Para cada valor de torque foram verificados os valores de carga através da célula de carga e valores do comprimento do parafuso através do equipamento de ultrassom. Como o parafuso foi submetido a torque, cargas de tração eram esperadas no mesmo e, por consequência, um aumento do comprimento do parafuso também.

método de ensaio proposto buscou determinar valores de carga e comprimento do parafuso ao passo que foi submetido a graduais níveis de torque. A Equação I foi utilizada para se calcular a força a partir do torque aplicado, sendo empregada para se obter os resultados do chamado método do torque. Após isso, utilizou-se a Equação 2 para obter os respectivos valores de tensão considerando a área da secção transversal do parafuso.

Por outro lado, tendo em mãos os valores da variação do comprimento do parafuso (alongamento) obtidos por ultrassom, utilizou-se a Equação 3 para se obter a força a partir do alongamento. Posteriormente, novamente a Equação 2 foi empregada para se converter estes valores de força em tensão.

$$
\begin{aligned}
& \mathrm{T}=\mathrm{FKD} \\
& \sigma=\mathrm{F} / \mathrm{A} \\
& \delta=\mathrm{FL} / \mathrm{AE}
\end{aligned}
$$

Onde:

T: Torque (Nm)

F: Força Tensora (N)

K: Fator de Torque

D: Diâmetro Nominal do Parafuso (m)

$\sigma:$ Tensão (MPa)

A: Área da Secção Transversal do Parafuso $\left(\mathrm{m}^{2}\right)$

$\delta$ : Alongamento $(\mathrm{m})$

L: Comprimento Inicial do Parafuso (m)

E: Módulo de Elasticidade (GPa)

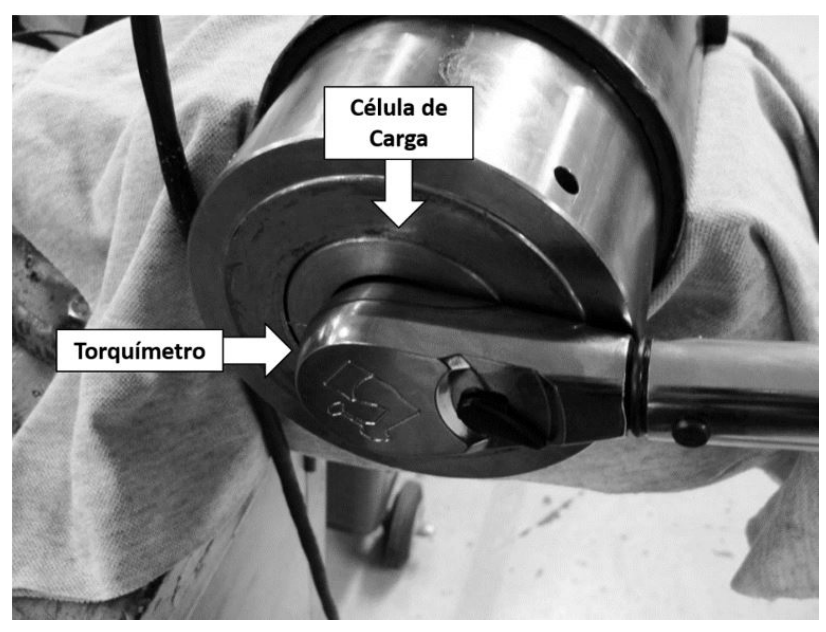

Figura I. Torquímetro acoplado junto à cabeça do parafuso para promover o torque. O parafuso já se encontra fixado dentro da célula de carga.

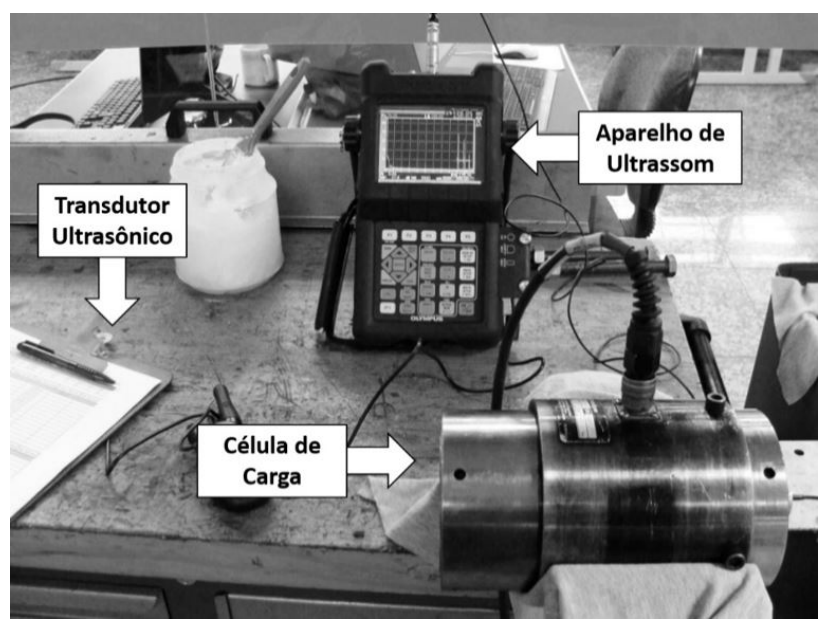

Figura 2. Montagem final de todo aparato experimental: célula de carga com os dispositivos para o ensaio e o aparelho de ultrassom utilizado.

\section{RESULTADOS E DISCUSSÕES}

Como já mencionado no capítulo 2, para relacionar o torque aplicado ao parafuso com o seu consequente aperto foi utilizado um torquímetro de estalo. Como os valores de torque são pré-estabelecidos em sua escala, atingindo-se estes determinados valores fazia-se a leitura da carga na célula de carga, chamada então de carga efetiva. Para efeitos 
comparativos, estes valores de carga foram convertidos para tensão através da Equação 2, e chamados de tensão efetiva.

A Figura 3 mostra um gráfico representado pelos valores de carga efetiva com relação a cada valor de torque aplicado, em cada um dos quatro parafusos ensaiados. Notam-se valores próximos de carga efetiva para cada parafuso ensaiado, indicando ser bastante reprodutivo o ensaio.

Já a Figura 4 mostra a média obtida através dos quatro parafusos para a tensão efetiva versus 0 torque aplicado. Observa-se que para tensões entre $100 \mathrm{MPa}$ e $350 \mathrm{MPa}$ o comportamento é linear, o que já era esperado sabendo da linearidade entre essas duas variáveis dentro do regime elástico comandado pela lei de Hooke $(3,4)$. Tal comportamento linear, porém, não é visto para tensões abaixo de $100 \mathrm{MPa}$, fato que pode estar relacionado com relaxação e alinhamento do parafuso(4). Tampouco para tensões acima de $350 \mathrm{MPa}$ o comportamento é linear, o que pode estar relacionado com o limite de escoamento do parafuso que é da ordem de $400 \mathrm{MPa}$ segundo a norma ISO 898-I:20 I 3 [I I] e sua consequente deformação plástica.

A Figura 5 mostra as curvas tensão versus deformação para as três formas de tensão: efetiva, estimada com base no torque e estimada com base no ultrassom. Verifica-se que a tensão com base no ultrassom se assemelha bastante com à tensão efetiva considerando a faixa de interesse que envolve a região elástica da curva, desde aproximadamente $100 \mathrm{MPa}$ até $350 \mathrm{MPa}$. É possível observar que até uma tensão aproximada de $100 \mathrm{MPa}$, não existe proporcionalidade, logo, essa região não pode ser levada em consideração. O mesmo fenômeno ocorreu nas curvas relacionadas ao torque, provavelmente relacionado às mesmas causas citadas anteriormente. Na região em torno de $400 \mathrm{MPa}$, é possível observar o início do comportamento plástico, indicando que o parafuso alcançou seu limite de escoamento.

A Figura 6 apresenta o erro relativo de cada uma das duas tensões estimadas frente à tensão efetiva. Analisando os resultados do cálculo da tensão com relação aos valores de torque aplicado, observou-se grande dispersão dos resultados, inclusive na região de interesse dos parafusos. Valores entre $12 \%$ a $30 \%$ (+48 MPa e - I $20 \mathrm{MPa}$ ) de erro relativo em comparação a tensão efetiva da célula de carga foram encontrados na região elástica do parafuso, excluindo-se tensões abaixo de $100 \mathrm{MPa}$. Isso vai ao encontro com o que foi mencionado no capítulo I deste trabalho, onde o erro encontrado para o controle da pré-carga via torque encontra-se ao redor de $20 \%$ [ $I]$.

Sob o ponto de vista do alongamento, calculando-se os valores de tensão através de medidas feitas por ultrassom, obteve-se valores da ordem de $0 \%$ até $8 \%(0 \mathrm{MPa}$ e $+32 \mathrm{MPa})$ de erro relativo em comparação com a tensão efetiva aplicada no parafuso. Para tais valores de erros, apenas a região elástica foi considerada e a região abaixo de $100 \mathrm{MPa}$ desconsiderada.

Como mencionado anteriormente, o alto nível de erro associado ao método do controle da pré-carga realizado

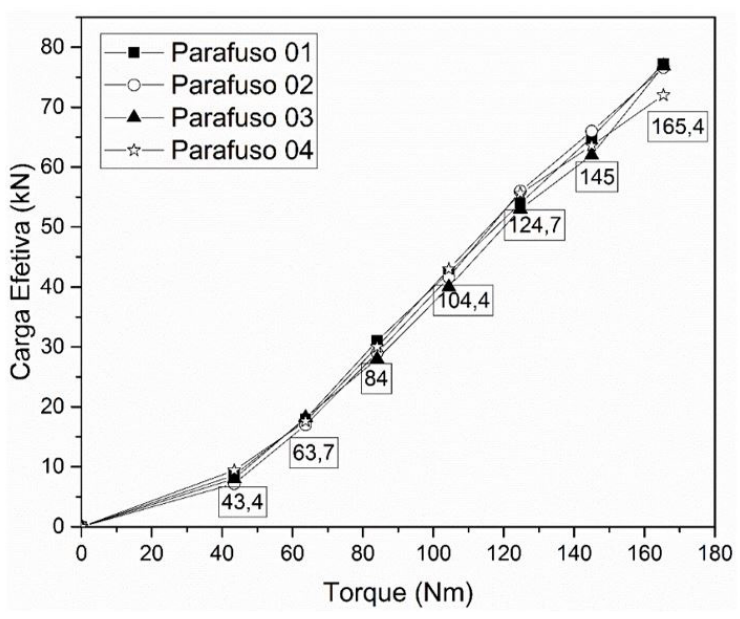

Figura 3. Valores de carga efetiva versus torque aplicado para cada parafuso ensaiado.

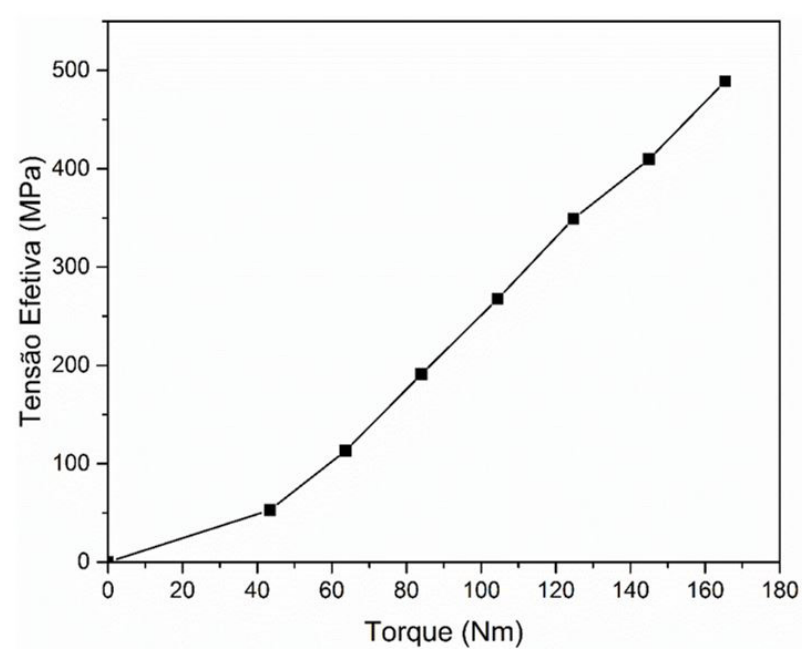

Figura 4. Tensão efetiva média para os quatro parafusos em função do torque.

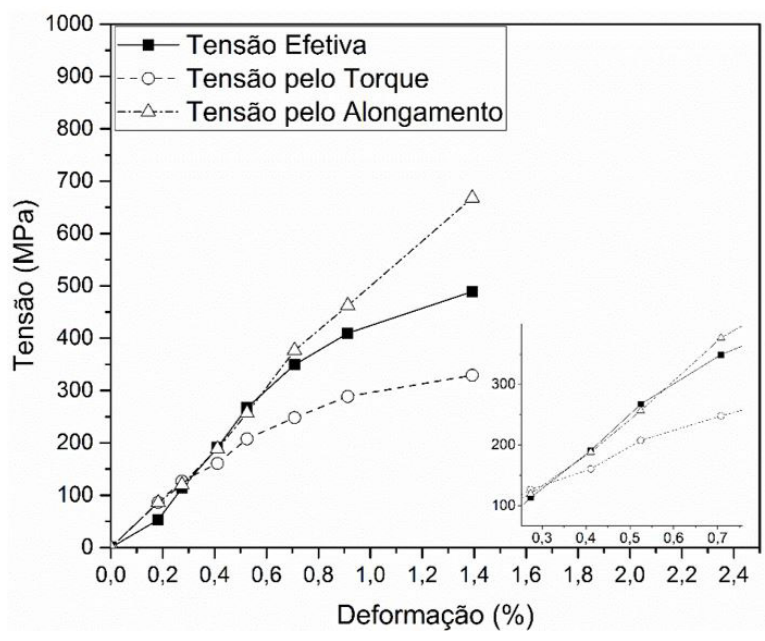

Figura 5. Curva tensão versus deformação para os dois métodos de controle da pré-carga. 


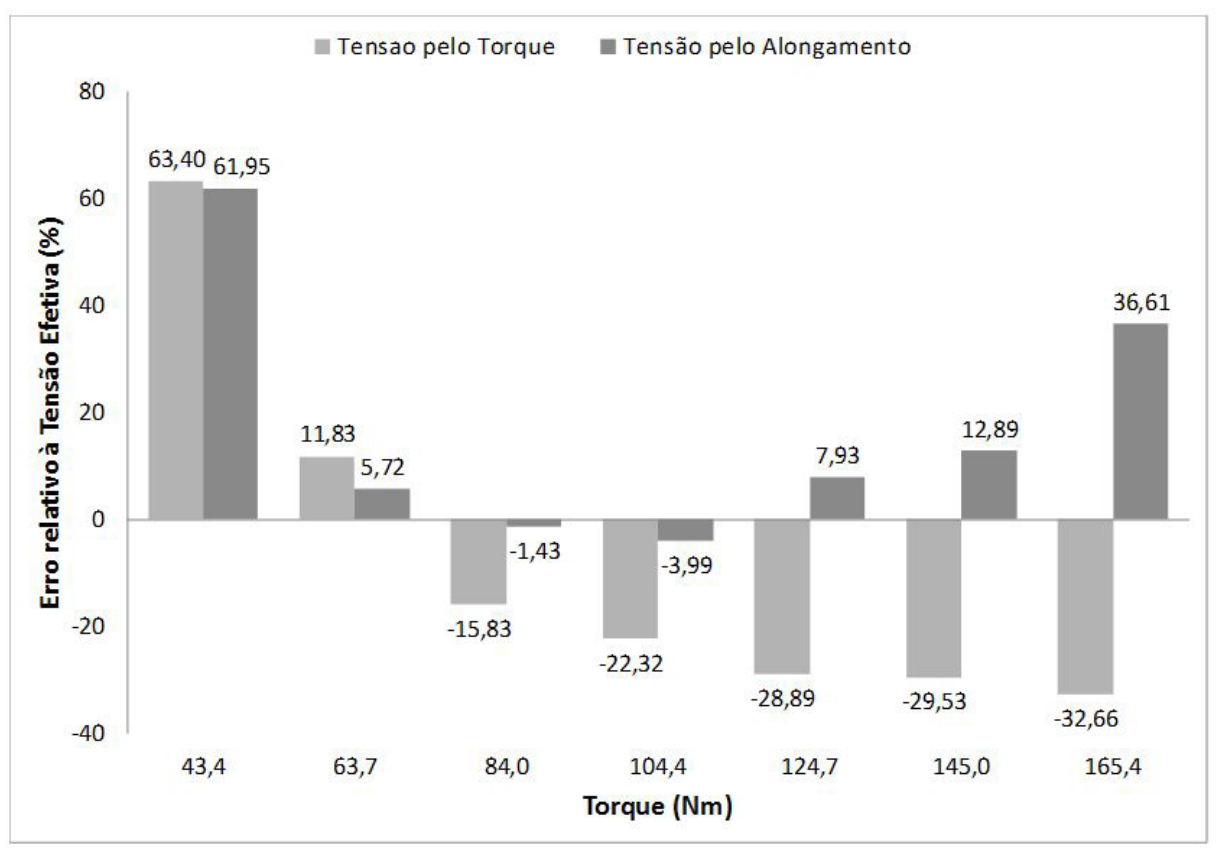

Figura 6. Erro relativo associado a cada uma das tensões estimadas frente à tensão efetiva.

diretamente pelo torque está diretamente associado às inúmeras variáveis presentes quando se comparam diretamente força e torque. A Equação I citada anteriormente apresenta uma relação diretamente proporcional entre o torque e o fator de torque $(\mathrm{K})$. Esta constante depende de fatores muito difíceis de serem estimados e altamente sujeitos a erros, tais como o coeficiente de atrito da cabeça e da rosca do parafuso bem como irregularidades dimensionais, defeitos nas roscas, sujidade, condições de lubrificação e aspecto superficial dos elementos envolvidos, etc [9].

Logo, por mais que existam tentativas de se diminuir o efeito do valor de $\mathrm{K}$ na Equação I utilizada para realizar o controle da pré-carga através do torque, o problema ainda está longe de ser superado. Uma das tentativas de minimização vem da norma ISO 16047:2005 [12] na qual utiliza o fator de torque através da discriminação dos valores do passo de rosca, diâmetro interno e médio do parafuso. Porém, continuam a existir diversos fatores de difícil controle como condições particulares de cada parafuso bem como da junta. Não esquecendo dos coeficientes de atrito, que continuam como sendo as principais fontes de erro uma vez que é praticamente impossível de mensurar os valores reais.

Ainda no método do controle do aperto pelo torque, existe uma perda significativa da tensão axial requerida em tensão de torção. Alguns autores citam que esta reação parasita possa consumir até $30 \%$ da tensão que o parafuso deveria sofrer, fazendo com que haja uma diminuição nos valores de pré-carga da junta(3,5,6,9). A Figura 5 mostra exatamente esta tendência de queda da tensão calculada pelo torque quando comparada a tensão efetiva medida pela célula de carga. A perda de carga por torção pode estar ocorrendo no sistema estudado neste trabalho.
Já realizando o controle da pré-carga diretamente pelo alongamento através da utilização da Equação 3 já mencionada, erros muito menores foram verificados na faixa de tensões consideradas como de interesse. Isso se deve ao fato de que as variáveis da Equação 3 são de fácil controle e muito menos subjetivas que o fator de torque, por exemplo. A fórmula engloba, entre outros fatores, o módulo de elasticidade - grandeza de pouca variação em se tratando de aços - e também o comprimento inicial do parafuso, facilmente mensurável. Leva-se em conta também que a medida ultrassônica fornece o alongamento como resultado final e com uma margem de erro muito baixa, parâmetro que também é considerado na Equação 3.

As considerações apenas da região elástica para a discussão dos resultados obtidos neste trabalho vão ao encontro do que já foi publicado até agora, onde a tensão de aperto de um parafuso deve permanecer na região elástica $[1-3,9]$. Portanto, para efeitos de comparação o método da medição por ultrassom do alongamento do parafuso se torna válido.

Uma vez adotado este método por setores vitais da indústria, acredita-se que haveria uma queda significativa no número de falhas em juntas parafusadas, principalmente aquelas relacionadas por problemas de montagem e que possam acarretar falhas por fadiga, responsáveis pela grande maioria de problemas atualmente. Somado a isso, como se viu neste trabalho o erro da tensão com base no torque em comparação com a tensão efetiva sofrida pelo parafuso é quase sempre para menos, ou seja, na vida real a união parafusada controlada pelo método do torque estaria sob uma tensão menor da qual foi projetada, sendo propensa a falhar por fadiga diminuindo consideravelmente a vida útil do equipamento no qual a junta estaria empregada. 


\section{CONCLUSÕES}

A análise dos resultados obtidos neste trabalho permitiu as seguintes conclusões:

a) Comparando-se os valores das tensões estimadas com base nas medidas das tensões efetivas pela célula de carga, os erros de medição da tensão calculada pelo método do torque variaram entre $+48 \mathrm{MPa} e$ - $120 \mathrm{MPa}$, enquanto os erros de medição da tensão calculada pelo alongamento medido por ultrassom variaram entre $0 \mathrm{MPa}$ e $+32 \mathrm{MPa}$; b) O alongamento medido por ultrassom possibilita a aplicação de uma carga mais precisa de aperto do parafuso que a utilização de torquímetros de aperto convencionalmente utilizados, permitindo com isso a montagem de uma união mais segura;

c) O controle do torque baseado no método da medição do alongamento por ultrassom somente se torna efetivo na região elástica do parafuso. Atingindo-se a região plástica, este método se torna ineficiente e passível de erro.

\section{REFERÊNCIAS}

I Fatemi A. Metal fatigue in engineering. 2nd ed. New York: Wiley-Interscience; 2000. 472 p.

2 Suresh S. Fatigue of Materials. 2nd ed. Cambridge: Cambridge University Press; 1998. 704 p. (Cambridge Solid State Science Series).

3 Budynas R, Nisbett K. Shigley's mechanical engineering design. 10th ed. New York: McGraw-Hill Education; 2014. $1104 \mathrm{p}$.

4 Shoberg RS. Engineering fundamentals of threaded fastener design and analysis. I. Fastening. 2000;6(2):26-29.

5 Blake A. Design of mechanical joints. New York: CRC Press; 1985. 562 p.

6 Juvinall RC, Marshek KM. Fundamentals of machine component design. 5th ed. Hoboken: Wiley; 201 I. 928 p.

7 Olaru DN, Bălan MRD, Tufescu A, Cârlescu V, Prisacaru G. Influence of the cage on the friction torque in low loaded thrust ball bearings operating in lubricated conditions. Tribology International. 2017;107:294-305.

8 Guo Y, Lambert S, Wallen R, Errichello R, Keller J. Theoretical and experimental study on gear-coupling contact and loads considering misalignment, torque, and friction influences. Mechanism and Machine Theory. 2016;98:242-262.

9 Bickford J. An introduction to the design and behavior of bolted joints. 3rd ed. New York: Marcel Dekker; 1995. $951 \mathrm{p}$.

10 Vallery H, Ekkelenkamp R, Van Der Kooij H, Buss M. Passive and accurate torque control of series elastic actuators. In: 2007 IEEE/RSJ International Conference on Intelligent Robots and Systems; 2007. San Diego, CA. Canada: IEEE; 2007.

II International Organization for Standardization - ISO. ISO 898-I:20I3 - Mechanical properties of fasteners made of carbon steel and alloy steel -- Part I: Bolts, screws and studs with specified property classes -Coarse thread and fine pitch thread. Genebra: ISO; 2013.

12 International Organization for Standardization - ISO. ISO 16047:2005 - Fasteners -- Torque/clamp force testing. Genebra: ISO; 2005.

Recebido em: 27 Jan. 2017

Aceito em: 12 Maio 2017 